Molecular dynamics of half-integer quadrupolar nuclei studied by QCPMG solid-state NMR experiments on static and rotating samples. Theory and simulations.

Hofmann Larsen, Flemming

Published in:

Journal of Magnetic Resonance

DOI:

10.1016/j.jmr.2004.09.006

Publication date:

2004

Document version

Early version, also known as pre-print

Citation for published version (APA):

Hofmann Larsen, F. (2004). Molecular dynamics of half-integer quadrupolar nuclei studied by QCPMG solid-

state NMR experiments on static and rotating samples. Theory and simulations. Journal of Magnetic Resonance, 171(2), 293-304. https://doi.org/10.1016/j.jmr.2004.09.006 


\title{
Molecular dynamics of half-integer quadrupolar nuclei studied by QCPMG solid-state NMR experiments on static and rotating samples. Theory and simulations
}

\author{
Flemming H. Larsen \\ Department of Protein Chemistry, Institute of Molecular Biology, University of Copenhagen, Øster Farimagsgade 2A, \\ DK-1353 Copenhagen K, Denmark
}

Received 2 July 2004; revised 10 September 2004

Available online 8 October 2004

\begin{abstract}
Simulations of QCPMG NMR type experiments have been used to explore dynamic processes of half-integer quadrupolar nuclei in solids. By setting up a theoretical approach that is well suited for efficient numerical simulations the QCPMG type experiments have been analyzed regarding the effect of the magnitude of the EFG- and CSA-tensors, the spin-quantum number, different dynamical processes and MAS. Compared to the QE experiment the QCPMG experiment offers not only intensity gain by an order of magnitude and changes in overall lineshape as a function of the kinetic rate constant but the lineshape of the individual spin-echo sidebands is also very sensitive towards dynamics. Hereby a visual identification of the dynamics is obtained. In common for all the simulations the spin-echo sidebands are narrow in the slow $\left(k \leqslant 10^{2} \mathrm{~Hz}\right)$ and the fast $\left(k \geqslant 10^{7} \mathrm{~Hz}\right)$ dynamic regime whereas they are broadened in the intermediate regime $10^{3} \leqslant k \leqslant 10^{7} \mathrm{~Hz}$. The maximum intensity of the spin-echo sidebands for two-site jumps is highly dependent on the type of anisotropic interactions involved and the type of QCPMG experiment. Hence, in the fast limit the maximum intensity was $140 \%$ of the initial intensity when significant CSA was present or under the QCPMG-MAS experiment compared to 89 or $71 \%$ for the static experiment influenced by the quadrupolar interaction only. For 3-, 4-, and 6-site jumps the maximum intensity in the fast limit reached up to $339 \%$ of the intensity in the static limit.
\end{abstract}

(C) 2004 Elsevier Inc. All rights reserved.

Keywords: QCPMG; Solid-state; N-site jump processes; Low- $\gamma$ quadrupolar nuclei; Simulations

\section{Introduction}

The major part of the NMR active nuclei in the periodic table are half-integer quadrupolar nuclei. Depending on their Larmor frequency, natural abundance and quadrupole moment these can be classified experimentally as easy, intermediate or difficult accessible nuclei. Recent advents of magnets with a very high magnetic field (up to $21.14 \mathrm{~T}$ ) have moved a wide range of low- $\gamma$ nuclei from the difficult into the intermediate range. Combined with improvements on probes and consoles this allows for more advanced experiments on these nu-

E-mail address: fhl@apk.molbio.ku.dk. clei. In this context studies of dynamics have become feasible for a wide range of nuclei such as ${ }^{39} \mathrm{~K},{ }^{25} \mathrm{Mg}$, ${ }^{67} \mathrm{Zn}$, and ${ }^{87} \mathrm{Sr}$, that previously have been difficult to study using state-of-the-art equipment.

Many of these nuclei are of particular interest because of their structural significance in ion-exchange materials [1-3], modifier ions in glasses [4], or active sites in metallo-proteins [5-8]. Therefore, experimental analysis of the dynamic properties for these nuclei is a key issue in order to gain important insight into these classes of materials and molecules. Traditionally studies of dynamics by solid-state NMR have been explored by ${ }^{2} \mathrm{H}$ NMR using either static or MAS experiments. This approach has proved to be a powerful tool for analysis 
of motional processes and significant effort has been devoted to analysis of deuterated compounds and development of experiments for comprehensive studies of ${ }^{2} \mathrm{H}$ dynamics [9-12].

Only recently the effects of molecular dynamics have been studied in detail for half-integer quadrupolar nuclei [13-16]. These studies have been performed using the either single-pulse, spin-echo or inversion recovery experiments under static or MAS conditions. Combined with lineshape simulations this has provided information about the dynamics.

Previously it has been demonstrated that static ${ }^{2} \mathrm{H}$ QCPMG experiments provided not only an increase in sensitivity but also a visible effect of the dynamics as the lineshape of the spin-echo sidebands is sensitive to the magnitude of the rate constant [17]. Therefore, the present work is focused on the effects of the QCPMG experiment [18] on the central transition under the influence of motion. The analysis will be performed for static QCPMG as well as QCPMG-MAS $[19,20]$ type experiments to explore effects of sample spinning.

\section{Theory}

The calculations presented here are based on the density operator formalism using the Liouville-von-Neumann equation. Following the approach of Barbara et al. [21] the Hamiltonian for a $N$-site jump may be written as

$\dot{\rho}=i[\rho, H]+k(\mathbf{P} \rho \mathbf{P}-\rho)$,

where $\rho$ is the density operator, $H$ is the effective Hamiltonian, $k$ is the exchange rate and $\mathbf{P}$ is a permutation operator. The density operator can be expressed as a linear combination of operators forming a complete basis set. For a spin- $I$ nucleus a complete basis set consists of $\operatorname{dim}=\left((2 I+1)^{2}-1\right)$ basis operators for each of the $N$ sites resulting in a total of $R=N \cdot \operatorname{dim}$ operators. Therefore, the density operator may be written as

$\rho(t)=\sum_{i=1}^{R} m_{i}(t) \mathbf{O}_{i}$,

where $\left\{\mathbf{O}_{i}\right\}_{i=1}^{R}$ denotes a complete set of basis operators and $m_{i}(t)$ denotes the time dependent coefficients $m_{i}(t)$, $i=1, \ldots, R$. Using the form of Eq. (1) these coefficients must fulfill

$\dot{m}_{i}=\sum_{j=1}^{R} L_{i j} m_{j}$

which has the general solution

$\vec{M}\left(t_{1}\right)=T \exp \left(\int_{t_{0}}^{t_{1}} L(t) \mathrm{d} t\right) \vec{M}\left(t_{0}\right)$,

where the Dyson time-ordering operator [22], $T$, is used for evaluating the integral when $L(t)$ is time depen- dent - e.g., under MAS. The exact expression for of $L(t)$ will be developed in the following.

The theoretical approach by Barbara et al. [21] only considers the case for $I=1$ and a two-site jump. Therefore, the basis set in their analysis has to be expanded in order to extend the formalism to include 3-, 4-, and 6-site jumps and spin quantum numbers $3 / 2,5 / 2,7 / 2$, and $9 / 2$ as well. An adequate basis set may be obtained from Bowden et al. $[23,24]$ which was developed for multiple-quantum experiments. In this formalism the irreducible spin operators $T_{q}^{n}, n=0, \ldots, 2 I ; q=-n, \ldots, n$, of rank $n$ and order $q$ form an orthogonal basis set. For general spin- $I$ the operators, $T_{q}^{n}$, are defined as [23]:

$$
\begin{aligned}
\left\langle I m\left|T_{q}^{n}\right| I m^{\prime}\right\rangle & \\
= & (-1)^{I-m}\left(\begin{array}{ccc}
I & n & I \\
-m & q & m^{\prime}
\end{array}\right)\left\langle I\left\|T^{n}\right\| I\right\rangle \\
= & (-1)^{2 I-n-m-m^{\prime}} \delta_{(-m+q),-m^{\prime}} \\
& \times n ! \sqrt{\frac{(I-m) !(I+m) !(n+q) !(n-q) !\left(I-m^{\prime}\right) !\left(I+m^{\prime}\right) !}{2^{n}(2 n !)}} \\
& \cdot \sum_{v=0}^{2 I} \frac{(-1)^{v}}{(I+m-v) !(I-n-m+v) !(n+q-v) !(v-q) ! v !(n-v) !},
\end{aligned}
$$

where the sum over $v$ is only defined when none of the terms contain negative factorials [25] and

$\left\langle I\left\|T^{n}\right\| I\right\rangle=\sqrt{\frac{n ! n !(2 I+n+1) !}{2^{n}(2 n) !(2 I-n) !}}$.

The orthonormal basis derived from these operators is going to form the complete set of basis operators. These are defined as [23]

$\hat{T}_{q}^{n}=\frac{1}{n !} \sqrt{\frac{(2 n+1)(2 I-n) ! 2^{n}(2 n !)}{(2 I+n+1) !}} T_{q}^{n}=\hat{C}_{I}^{n} T_{q}^{n}$.

The theoretical approach will take use of these operators. The effective Hamiltonian will be described for a single site, site $v$, but for simplicity the formalism omits the index $v$ unless it is absolutely necessary as the expressions are equivalent for all sites in the $N$-site jump process. For a system including both CSA- and quadrupolar interactions the effective Hamiltonian for a single site during a pulse is

$H_{\text {tot }}=H_{Q}^{(1)}+H_{Q}^{(2)}+H_{\sigma}+H_{\mathrm{rf}}$,

in which $H_{\sigma}$ includes the isotropic as well as the anisotropic part of the chemical shielding, and $H_{Q}^{(1)}$ and $H_{Q}^{(2)}$ denote the first order and the secular parts of the second order quadrupolar interaction, respectively, and $H_{\mathrm{rf}}$ represents the rf-operator for a pulse with phase $\theta$. In the standard Zeeman basis these operators are defined as

$H_{Q}^{(1)}=\omega_{Q}^{(1)} \frac{1}{\sqrt{6}}\left(3 I_{z}^{2}-I(I+1)\right)$, 


$$
\begin{aligned}
H_{Q}^{(2)}= & \omega_{Q}^{(21)}\left(-8 I_{z}^{3}+4 I(I+1) I_{z}-I_{z}\right) \\
& +\omega_{Q}^{(22)}\left(-2 I_{z}^{3}+2 I(I+1) I_{z}-I_{z}\right), \\
H_{\sigma}=- & \left(\sqrt{\frac{2}{3}} \omega_{\sigma}+\omega_{\mathrm{iso}}\right) I_{z}, \\
H_{\mathrm{rf}}= & -\omega_{\mathrm{rf}}\left(I_{x} \cos (\theta)+I_{y} \sin (\theta)\right),
\end{aligned}
$$

where

$$
\omega_{Q}^{(1)}=\omega_{q} R_{L}^{Q}(0)
$$

$\omega_{Q}^{(2 j)}=-\frac{\omega_{q}^{2}}{2 \omega_{0}} R_{L}^{Q}(j) R_{L}^{Q}(-j)$,

$\omega_{q}=\frac{2 \pi C_{Q}}{2 I(2 I-1)}$,

$\omega_{\sigma}=\omega_{0} R_{L}^{\sigma}(0)$,

$\omega_{\text {iso }}=\delta_{\text {iso }} \omega_{0}$,

and $C_{Q}=\frac{\mathrm{e}^{2} q Q}{h}$ denotes the quadrupolar coupling constant, $\omega_{\mathrm{rf}}=-\gamma \mathbf{B}_{\mathrm{rf}}$ is the rf-field strength, $\omega_{0}=-\gamma \mathbf{B}_{0}$ the Larmor frequency and $\delta_{\text {iso }}$ and $\delta_{\sigma}$ the isotropic and anisotropic chemical shift (CSA), respectively. In their principal axis frames $(\mathrm{P})$ the non-zero elements of the EFG (superscript $Q$ )- and CSA (superscript $\sigma$ )tensors are given by $R_{P}^{Q}(-2)=R_{P}^{Q}(2)=-\eta_{Q} / 2, R_{P}^{Q}(0)=$ $\sqrt{\frac{3}{2}}, \quad$ and $\quad R_{P}^{\sigma}(-2)=R_{P}^{\sigma}(2)=-\delta_{\sigma} \eta_{\sigma} / 2, \quad R_{P}^{\sigma}(0)=\sqrt{\frac{3}{2}} \delta_{\sigma}$, respectively. The spatial parts of the tensors in the laboratory frame (L) for the $v^{\prime}$ th site are calculated as

$$
\begin{aligned}
R_{L}^{Q}(n)= & \sum_{m=-2}^{2} \sum_{k=-2}^{2} \sum_{j=-2}^{2} R_{P}^{Q}(j) \mathbf{D}_{j, k}^{(2)}\left(\alpha_{P C, v}^{Q}, \beta_{P C, v}^{Q}, \gamma_{P C, v}^{Q}\right) \\
& \times \mathbf{D}_{k, m}^{(2)}\left(\alpha_{C R}, \beta_{C R}, \gamma_{C R}\right) \mathbf{D}_{m, n}^{(2)}\left(\omega_{r} t, \beta_{R L}, 0\right),
\end{aligned}
$$

$$
\begin{aligned}
R_{L}^{\sigma}(0)= & \sum_{m=-2}^{2} \sum_{k=-2}^{2} \sum_{q=-2}^{2} \sum_{j=-2}^{2} R_{P}^{\sigma}(j) \mathbf{D}_{j, q}^{(2)}\left(\alpha_{P P}^{\sigma}, \beta_{P P}^{\sigma}, \gamma_{P P}^{\sigma}\right) \\
& \times \mathbf{D}_{q, k}^{(2)}\left(\alpha_{P C, v}^{Q}, \beta_{P C, v}^{Q}, \gamma_{P C, v}^{Q}\right) \mathbf{D}_{k, m}^{(2)}\left(\alpha_{C R}, \beta_{C R}, \gamma_{C R}\right) \\
& \times \mathbf{D}_{m, 0}^{(2)}\left(\omega_{r} t, \beta_{R L}, 0\right),
\end{aligned}
$$

where $\Omega_{P C, v}^{Q}=\left(\alpha_{P C, v}^{Q}, \beta_{P C, v}^{Q}, \gamma_{P C, v}^{Q}\right)$ are the angles describing the transformation from the principal axis frame $(\mathrm{P})$ of the EFG-tensor into the crystal fixed frame (C) for site $v$. It is noted that the Euler angles corresponding to a $N$-site jump may be given by [26]

$\Omega_{P C, v}^{Q}=\left(\theta, \phi, \frac{2 \pi(v-1)}{N}\right), \quad$ where $v \in\{1, \ldots, N\}$.

When the CSA is present the Euler angles $\Omega_{P P}^{\sigma}=\left(\alpha_{P P}^{\sigma}, \beta_{P P}^{\sigma}, \gamma_{P P}^{\sigma}\right)$ transform the CSA-tensor from its principal axis system into the principal axis system of the EFG-tensor. The angles for powder averaging
$\Omega_{C R}=\left(\alpha_{C R}, \beta_{C R}, \gamma_{C R}\right)$ describe the transformation from the crystal frame to the rotating frame $(\mathrm{R})$ in case of MAS. The last transformation by $\Omega_{C R}=\left(\omega_{r} t, \beta_{R L}, 0\right)$ describes the transformation from the rotating frame to the laboratory frame, where $\omega_{r}$ denotes the spin-rate and $\beta_{R L}$ the magic angle. For static samples Eqs. (18) and (19) are reduced to

$$
\begin{aligned}
R_{L}^{Q}(n)= & \sum_{k=-2}^{2} \sum_{j=-2}^{2} R_{P}^{Q}(j) \mathbf{D}_{j, k}^{(2)}\left(\Omega_{P C, v}^{Q}\right) \mathbf{D}_{k, n}^{(2)}\left(\alpha_{C R}, \beta_{C R}, 0\right), \\
R_{L}^{\sigma}(0)= & \sum_{k=-2}^{2} \sum_{q=-2}^{2} \sum_{j=-2}^{2} R_{P}^{Q}(j) \mathbf{D}_{j, q}^{(2)}\left(\Omega_{P P}^{\sigma}\right) \\
& \times \mathbf{D}_{q, k}^{(2)}\left(\Omega_{P C, v}^{Q}\right) \mathbf{D}_{k, 0}^{(2)}\left(\alpha_{C R}, \beta_{C R}, 0\right) .
\end{aligned}
$$

Using the tensor operators $T_{q}^{n}$ the Hamiltonians may be described as

$$
\begin{aligned}
H_{Q}^{(1)}= & \omega_{Q}^{(1)} T_{0}^{2}, \\
H_{Q}^{(2)}= & \omega_{Q}^{(21)}\left(-8 \sqrt{\frac{2}{5}} T_{0}^{3}-\left(\frac{4}{5} I(I+1)-\frac{3}{5}\right) T_{0}^{1}\right) \\
& +\omega_{Q}^{(22)}\left(-2 \sqrt{\frac{2}{5}} T_{0}^{3}+\left(\frac{4}{5} I(I+1)-\frac{3}{5}\right) T_{0}^{1}\right) \\
= & \sqrt{\frac{2}{5}}\left(-8 \omega_{Q}^{(21)}-2 \omega_{Q}^{(22)}\right) T_{0}^{3} \\
& -\left(\frac{4}{5} I(I+1)-\frac{3}{5}\right)\left(\omega_{Q}^{(21)}-\omega_{Q}^{(22)}\right) T_{0}^{1}
\end{aligned}
$$

$H_{\sigma}=-\left(\sqrt{\frac{2}{3}} \omega_{\sigma}+\omega_{\text {iso }}\right) T_{0}^{1}$

$H_{\mathrm{rf}}=\frac{\omega_{\mathrm{rf}}}{\sqrt{2}}\left(\cos (\theta)\left(T_{1}^{1}-T_{-1}^{1}\right)-\mathrm{i} \sin (\theta)\left(T_{1}^{1}+T_{1}^{-1}\right)\right)$

That is, the total effective Hamiltonian during a pulse with phase $\theta$ may be written as

$$
\begin{aligned}
H_{\text {tot }}= & a T_{0}^{1}+b\left(\cos (\theta)\left(T_{1}^{1}-T_{-1}^{1}\right)-\mathrm{i} \sin (\theta)\left(T_{1}^{1}+T_{1}^{-1}\right)\right) \\
& +c T_{0}^{2}+d T_{0}^{3}=\frac{1}{\hat{C}_{I}^{1}}\left(a \hat{T}_{0}^{1}+b\left(\cos (\theta)\left(\hat{T}_{1}^{1}-\hat{T}_{-1}^{1}\right)\right.\right. \\
& \left.\left.-i \sin (\theta)\left(\hat{T}_{1}^{1}+\hat{T}_{1}^{-1}\right)\right)\right)+\frac{c}{\hat{C}_{I}^{2}} \hat{T}_{0}^{2}+\frac{d}{\hat{C}_{I}^{3}} \hat{T}_{0}^{3}
\end{aligned}
$$

where $a, b, c$, and $d$ are given by

$$
a=-\left(\omega_{\mathrm{iso}}+\sqrt{\frac{2}{3}} \omega_{\sigma}\right)+\frac{(4 I(I+1)-3)}{5}\left(\omega_{Q}^{(21)}-\omega_{Q}^{(22)}\right),
$$

$b=\sqrt{\frac{1}{2}} \omega_{\mathrm{rf}}$, 
$c=\omega_{Q}^{(1)}$,

$d=\frac{2 \sqrt{2}}{\sqrt{5}}\left(4 \omega_{Q}^{(21)}+\omega_{Q}^{(22)}\right)$.

During acquisition and delay periods the effective Hamiltonian reduces to

$H_{\mathrm{tot}}=\frac{a}{\hat{C}_{I}^{1}} \hat{T}_{0}^{1}+\frac{c}{\hat{C}_{I}^{2}} \hat{T}_{0}^{2}+\frac{d}{\hat{C}_{I}^{3}} \hat{T}_{0}^{3}$.

That is, only zero order operators are present during these periods. Arranging the basis operators according to their order, i.e.

$\left\{\hat{T}_{-2 I}^{2 I}, \hat{T}_{-2 I+1}^{2 I}, \hat{T}_{-2 I+1}^{2 I-1}, \ldots, \hat{T}_{2 I-1}^{2 I}, \hat{T}_{2 I-1}^{2 I-1}, \hat{T}_{2 I}^{2 I}\right\}$

and taking the form of Eq. (1) into account the $L$-matrix must be of the form

$L=\left[\begin{array}{cccc}A_{1}-(N-1) k E & \ldots & & k E \\ k E & A_{2}-(N-1) k E & \ldots & k E \\ \vdots & \vdots & \vdots & \vdots \\ k E & \ldots & k E & A_{N}-(N-1) k E\end{array}\right]$,

where $E$ is the identity matrix and the entries of $A_{v}$ may be calculated as:

$$
\begin{aligned}
A_{v}(m, n) & =\mathrm{i}\left\langle\left[\mathbf{O}_{m}, H_{t o t, v}\right], \mathbf{O}_{n}\right\rangle, v \in\{1, \ldots, N\} ; \\
m, n & \in\{1, \ldots, \operatorname{dim}\},
\end{aligned}
$$

where $H_{t o t, v}$ denotes the effective Hamiltonian for site $\mathrm{V}$. It is noted that the $A_{v}$-matrices and thereby the $L$-matrix during free precession are block-diagonal consisting of $1 \times 1, \ldots, 2 I \times 2 I$ matrices as different coherence orders do not mix when no rf-pulses are present. This means that the exponential of the $L$-matrix may be evaluated using $N \times N, \ldots,(2 N) I \times(2 N) I$ dimensional sub-spaces when dynamics is included for a $N$-site jump.

One specific submatrix, $L_{0}$, operating on the basis operators $\hat{T}_{0}^{2 I}, \ldots, \hat{T}_{0}^{1}$ for a $N$-site jump during free precession can be evaluated analytically because of its high degree of symmetry. That is,

$L_{0}=\left[\begin{array}{cccc}-(N-1) k E & \ldots & & k E \\ k E & -(N-1) k E & \ldots & k E \\ \vdots & & & \\ k E & k E & \ldots & -(N-1) k E\end{array}\right]$

which means that

$\exp \left(L_{0} t\right)=\frac{1}{N}\left[\begin{array}{cccc}X_{1} & X_{2} & \ldots & X_{2} \\ X_{2} & X_{1} & \ldots & X_{2} \\ \vdots & & & \\ X_{2} & \ldots & X_{2} & X_{1}\end{array}\right]$ in which

$X_{1}=\left(1+(N-1) \mathrm{e}^{-N k t}\right) E$,

$X_{2}=\left(1-\mathrm{e}^{-N k t}\right) E$.

The signal obtained for a single crystallite corresponding to the orientation $\Omega_{C R}=\left(\alpha_{C R}, \beta_{C R}, \gamma_{C R}\right)$ is calculated using:

$\operatorname{sig}\left(t, \Omega_{C R}\right)=\operatorname{Tr}\left(I_{+} \rho\left(t, \Omega_{C R}\right)\right)$.

That is, only parts of the density operator containing terms with $I_{-}$contributes to the signal. Noting that $I_{+}=-\sqrt{10} \hat{T}_{1}^{1}$ and using that the $\hat{T}_{q}^{n}$ operators form an orthonormal basis only $\hat{T}_{-1}^{1}$ contribute to the signal. Taking into account that

$\hat{T}_{-1}^{1}=-\left(\hat{T}_{1}^{1}\right)^{+}$,

$$
\begin{aligned}
\operatorname{Tr}\left(I_{+} \hat{T}_{-1}^{1}\right) & =-\sqrt{10} \operatorname{Tr}\left(\hat{T}_{1}^{1} \hat{T}_{-1}^{1}\right)=\sqrt{10} \operatorname{Tr}\left(\hat{T}_{1}^{1}\left(\hat{T}_{1}^{1}\right)^{+}\right) \\
& =\sqrt{10}
\end{aligned}
$$

the total powder signal for a $N$-site jump process may be calculated as:

$$
\begin{aligned}
\operatorname{sig}(t)= & \frac{\sqrt{10}}{8 \pi^{2}} \int_{0}^{2 \pi} \int_{0}^{\pi} \int_{0}^{2 \pi} \sum_{v=1}^{N} m_{-1, v}^{1}\left(t, \Omega_{C R}\right) \mathrm{d} \alpha_{C R} \\
& \times \sin \left(\beta_{C R}\right) \mathrm{d} \beta_{C R} \mathrm{~d} \gamma_{C R},
\end{aligned}
$$

where $m_{-1, v}^{1}\left(t, \Omega_{C R}\right)$ denotes the coefficient of $\hat{T}_{-1}^{1}$ for the $v^{\prime}$ th site.

\section{Numerical simulations}

Calculations were performed for nuclei having spins of $3 / 2,5 / 2,7 / 2$, and $9 / 2$. The Larmor frequencies were $23.325(I=3 / 2), 30.597(I=5 / 2), 33.641(I=7 / 2)$, and $39.004(I=9 / 2) \mathrm{MHz}$ when only the quadrupole interaction was included. This corresponds to ${ }^{39} \mathrm{~K},{ }^{25} \mathrm{Mg}$, ${ }^{43} \mathrm{Ca}$ at $11.74 \mathrm{~T}$, and ${ }^{87} \mathrm{Sr}$ at $21.14 \mathrm{~T}$. Regarding analysis of the CSA-effect on a spin-7/2-nucleus a Larmor frequency of $118.071 \mathrm{MHz}$ was employed corresponding to ${ }^{59} \mathrm{Co}$ at $11.74 \mathrm{~T}$.

The QCPMG/QCPMG-MAS pulse sequence may be depicted as

$$
\begin{aligned}
& \left(\frac{\pi}{2}\right)-\tau_{1}-(\pi)-\tau_{2}-\text { Acq. }\left(\frac{\tau_{a}}{2}\right)-\left[\tau_{3}-(\pi)-\tau_{4}\right. \\
& \left.- \text { Acq. }\left(\tau_{a}\right)\right]^{M}-\text { Acq. }\left(\tau_{d}\right),
\end{aligned}
$$

where $(\phi)$ denotes a central transition selective $\phi$-pulse, $\tau_{j}$ a delay and Acq. $(\tau)$ acquisition during a period $\tau$. For QCPMG-MAS the condition $2 p T_{r}=\tau_{3}+\tau_{4}+t_{\pi}+\tau_{a}$, where $p$ is an integer, $t_{\pi}$ is the duration of a selective $\pi$-pulse, and $T_{r}$ the rotor period, must be fulfilled.

Based on the previously observed significant effect of finite rf-pulses in QCPMG [18] and QCPMG-MAS [20] spectra this effect is included in all calculations. Unless stated specifically in the figure caption the following 
parameters were employed for the static QCPMG spectra: $\eta_{Q}=0.5, \delta_{\text {iso }}=0.0 \mathrm{ppm}, \tau_{a}=\tau_{d}=1.0 \mathrm{~ms}, M=40$, $\tau_{1}=\tau_{2}=\tau_{3}=\tau_{4}=100.0 \mu \mathrm{s}$, dwell time $=5.0 \mu \mathrm{s}$ using a Gaussian linebroadening of $10 \mathrm{~Hz}$. The same parameters were applied for the calculation of the QCPMGMAS spectra except for $\tau_{d}=0.5 \mathrm{~ms}, \quad M=20$, $\tau_{1}=\tau_{2}=50.0 \mu \mathrm{s}, \quad \tau_{3}=\tau_{4}=46.0 \mu \mathrm{s}$ and a Gaussian linebroadening of $20 \mathrm{~Hz}$. The spin-rate was $20.0 \mathrm{kHz}$ and the time increments were $1.0 \mu$ s during both rfpulses and periods of free precession.

For a $N$-site jump the Euler angles for the $v^{\prime}$ th site were defined by the orientations $\Omega_{P C, v}^{Q}=\left(0, \frac{\pi}{3}, v \frac{2 \pi}{N}\right)$, $v=0, \ldots,(N-1)$. Powder averaging was obtained by using $6765\left(\alpha_{C R}, \beta_{C R}\right)$ angle-pairs according to the Zaremba scheme [27] for the static spectra whereas 610 Zaremba $\left(\alpha_{C R}, \beta_{C R}\right)$ angle-pairs and 20 equidistantly distributed $\gamma_{C R}$-angles were employed for the QCPMG-MAS calculations.

The rf-field strengths were all in the central transition selective regime using the condition $\frac{2 \pi C_{Q}}{4 I(2 I-1)\left|\omega_{r}\right|}>3$. This means that the nominal pulse widths were divided by $(I+1 / 2)$.

All calculations were performed on a Dell Inspiron 8200 (Laptop) equipped with a $2.0 \mathrm{GHz}$ Pentium IV processor using homemade Fortran 77 programs. The programs employ the zgeev-routine from the LAPACK-package [28] for diagonalization and determination of eigenvalues and eigenvectors of matrices with complex entries. Processing times varied between $2.5 \mathrm{~min}$ (two-site jump, $I=3 / 2$ ) to $847 \mathrm{~min}$ (two-site jump, $I=9 / 2$ ) for the calculation of a static QCPMG FID whereas 2119 min were required for a QCPMGMAS FID (two-site jump, $I=3 / 2$ ). For comparison an ideal single-pulse MAS FID with 256 complex points using conditions identical to the QCPMG-MAS calculations a processing time of $50 \mathrm{~min}$. was required. This significantly longer processing time compared to a static QCPMG calculation is in part caused by the fact that the numerical integration needs to be performed for 3 Euler angles instead of 2 for a static. More importantly, the non-diagonal entries in the Hamiltonian during delays demand for time-ordered propagation in these periods which includes a diagonalization for each time increment whereas only one diagonalization is required for each delay in the static case.

\section{Results and discussion}

\section{1. $Q C P M G$ vs. $Q E$ experiments}

In Fig. 1A a range of calculated quadrupole echo (QE) $[29,30]$ and QCPMG spectra for a fictitious spin$3 / 2$ nucleus employing different kinetic rate constants are presented. All spectra are calculated for a two-site jump process. In the region of $k \leqslant 10^{2} \mathrm{~Hz}$ (Fig. 1A, a1 to Fig. 1A, c1) the lineshapes for the QE spectra are almost identical whereas a significant linebroadening and thereby drop in intensity is observed for the QCPMG spectra when going from $k=10^{1} \mathrm{~Hz}$ (Fig. 1A, b2) to $k=10^{2}$ (Fig. 1A, c2) Hz. For $k=10^{3} \mathrm{~Hz}$ (Fig. 1A, d2) broad lines are observed in the QCPMG spectrum whereas subtle changes in the lineshape are observed for the QE spectrum. In the intermediate regime, $10^{4} \leqslant k \leqslant 10^{7} \mathrm{~Hz}$, major changes in the lineshape are observed in both kinds of spectra but moreover the individual spin-echo sidebands become narrower in the QCPMG spectra. At faster dynamics minor changes in the lineshape are observed for both QE and QCPMG spectra but in the QCPMG spectra the spin-echo sidebands also narrow down to the same level as for very slow dynamics.

The relative maximum intensities for the $\mathrm{QE}$ and QCPMG spectra are shown in Fig. 1B. Because of the intensity gain by a factor of 43 due to the train of $\pi$-pulses the intensity of the QCPMG spectra are significantly stronger than the corresponding QE spectra. The trend observed by the linewidth as a function of $\log (k)$ is illustrated by the intensity minimum at $10^{3} \leqslant k \leqslant 10^{5} \mathrm{~Hz}$. Moreover it is noticed that the intensity descends faster than it rises and only reaches $71 \%$ of its initial magnitude in the fast dynamics limit.

\subsection{Effect of spin-quantum number}

To explore the effect of the spin-quantum number $I$ a series of QCPMG spectra are calculated for fictitious spin-3/2, spin-5/2, spin-7/2, and spin- $9 / 2$ nuclei performing two-site jumps. These spectra are displayed in Fig. 2. In general the spin-echo sidebands follows the previously described pattern for $I=3 / 2$ but for $I=9 / 2$ the lower rf-field strength reduces the intensity of the spin-echo sidebands around $8 \mathrm{kHz}$ in the spectra for $10^{5} \leqslant k \leqslant 10^{8} \mathrm{~Hz}$ (Fig. 2, f4-i4). The relative maximum intensities for the various nuclei as a function of rate constant are depicted in Fig. 3. For clarity the intensity of the spectra with $k=10^{-9} \mathrm{~Hz}$ (not shown) are set to 1.00 for all nuclei. It is noted that the intensity are almost identical for $k \leqslant 10^{3} \mathrm{~Hz}$. From this point it seems that the intensity increases at a slower rate for $I=7 / 2$ and $9 / 2$ than for $I=3 / 2$ and $5 / 2$. In the fast limit the intensities for $I=9 / 2$ is lower than the nuclei with lower spins. This is mainly ascribed to the lower rf-field strength because the spin-echo sidebands with the highest intensities are $12 \mathrm{kHz}$ away from the transmitter and therefore not ideally excited with an rf-fields strength of only $16.67 \mathrm{kHz}$.

\subsection{Effect of $C_{Q}$, spin-echo sideband separation and CSA}

To clarify whether the magnitude of $C_{Q}$, the spin-echo sideband separation and the CSA have any effect of the 
A

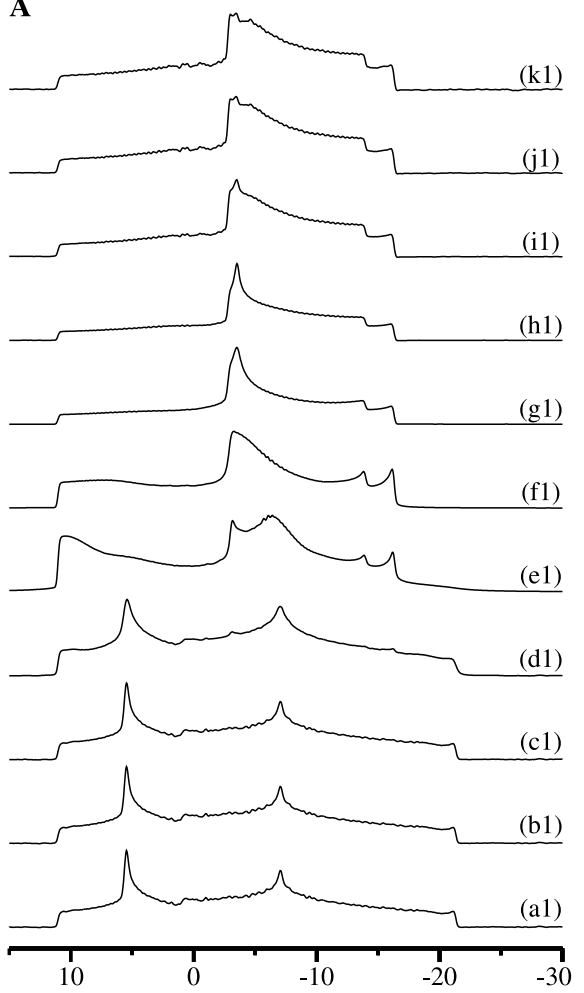

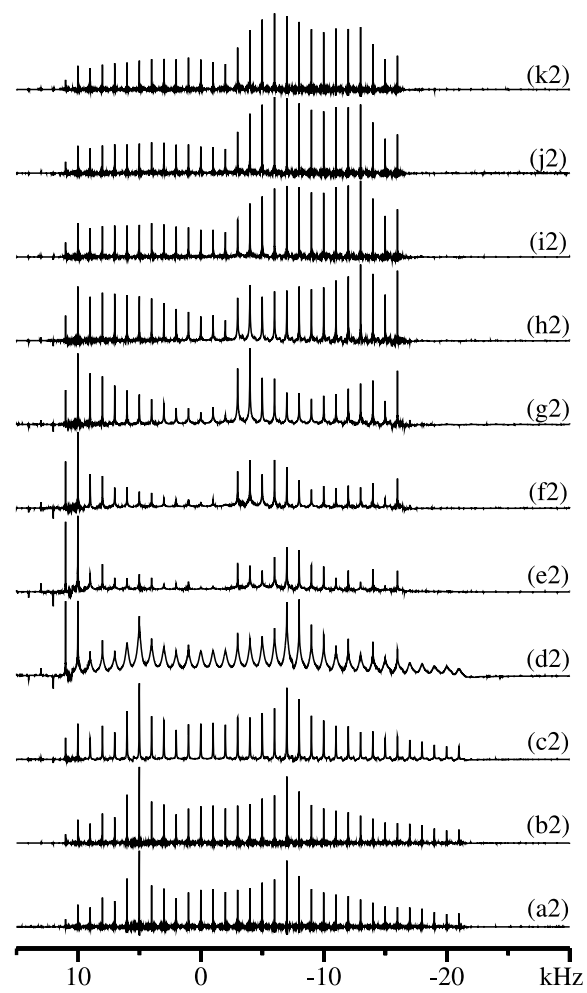

B

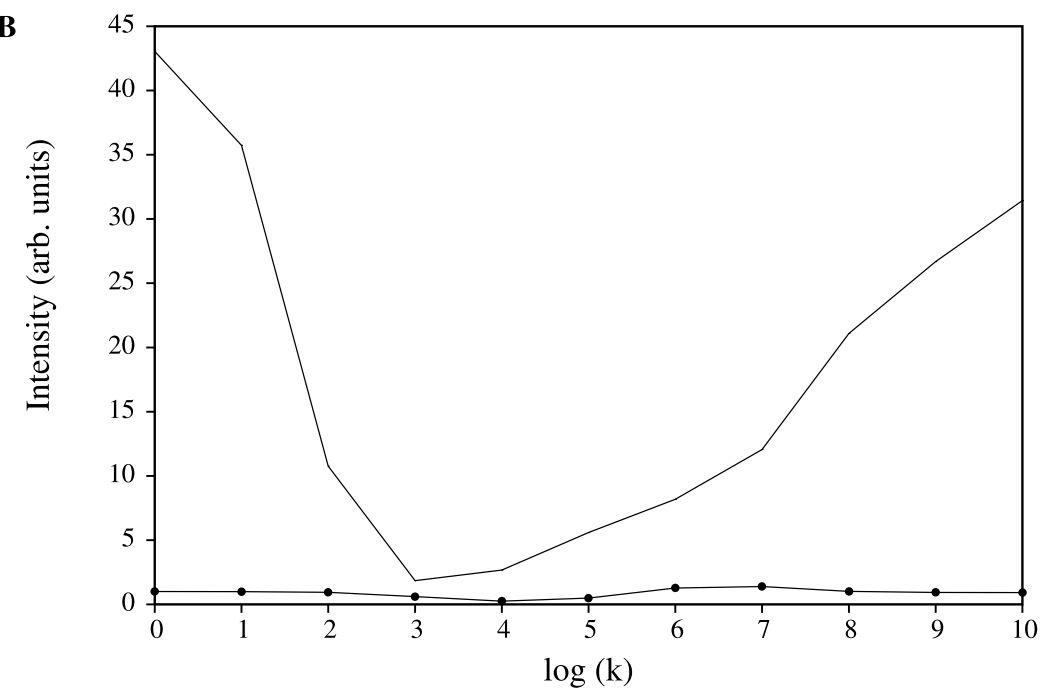

Fig. 1. (A) Calculated static spin-3/2 QE (left column) and QCPMG (right column) spectra for a two-site jump employing rate constants of (a1, a2) $10^{0}$, (b1, b2) $10^{1},(\mathrm{c} 1, \mathrm{c} 2) 10^{2},(\mathrm{~d} 1, \mathrm{~d} 2) 10^{3},(\mathrm{e} 1, \mathrm{e} 2) 10^{4},(\mathrm{f} 1, \mathrm{f} 2) 10^{5},(\mathrm{~g} 1, \mathrm{~g} 2) 10^{6},(\mathrm{~h} 1, \mathrm{~h} 2) 10^{7},(\mathrm{i} 1, \mathrm{i} 2) 10^{8},(\mathrm{j} 1, \mathrm{j} 2) 10^{9}$, and $(\mathrm{k} 1, \mathrm{k} 2) 10^{10} \mathrm{~Hz}$. The spectra are calculated using $\omega_{\mathrm{rf}}=-31.25 \mathrm{kHz}, C_{Q}=2.0 \mathrm{MHz}$ and apodized by Gaussian linebroadenings of 250 and $10 \mathrm{~Hz}$ for the QE and QCPMG spectra, respectively. For the QE spectra in the left column the relative intensity of the spectra are (a1) 1.00 , (b1) 0.99 , (c1) 0.94 , (d1) 0.61 , (e1) 0.25 , (f1) 0.49 , (g1) 1.27, (h1) 1.39, (i1) 1.01, (j1) 0.93, and (k1) 0.92, respectively. For the QCPMG spectra in the right column the relative intensity of the spectra are (a2) 0.98 , (b2) 0.81 , (c2) 0.25, (d2) 0.042, (e2) 0.061, (f2) 0.13, (g2) 0.19, (h2) 0.27, (i2) 0.48, (j2) 0.61, and (k2) 0.71, respectively. The maximum intensity of the spectrum in (a2) is 43.1 times higher than the spectrum in (a1). (B) Maximum intensities of calculated spin-3/2 QE (filled circles) and QCPMG (solid line) spectra. The intensities of the QE spectrum corresponding to $k=10^{-9} \mathrm{~Hz}$ (not shown) are set to 1.0 for reference.

intensity as a function of the rate constant a series of spectra varying these parameters have been calculated. Their corresponding maximum intensities are depicted in Fig. 4. In Fig. 4A maximum intensities for spin-3/2 QCPMG spectra calculated for $C_{Q}=2.0$ (solid line) and $4.0 \mathrm{MHz}$ (filled circles) are displayed. In the slow dynamics region below $k=10^{3} \mathrm{~Hz}$ the intensities agrees very well but the intensity minimum is broader for the larger $C_{Q}$ and only $53 \%$ of the initial magnitude is reached in the fast limit compared to $71 \%$ for the smaller $C_{Q}$.

Fig. 4B displays the intensity course for a series of spin-3/2 QCPMG spectra calculated for $C_{Q}=4.0 \mathrm{MHz}$ 


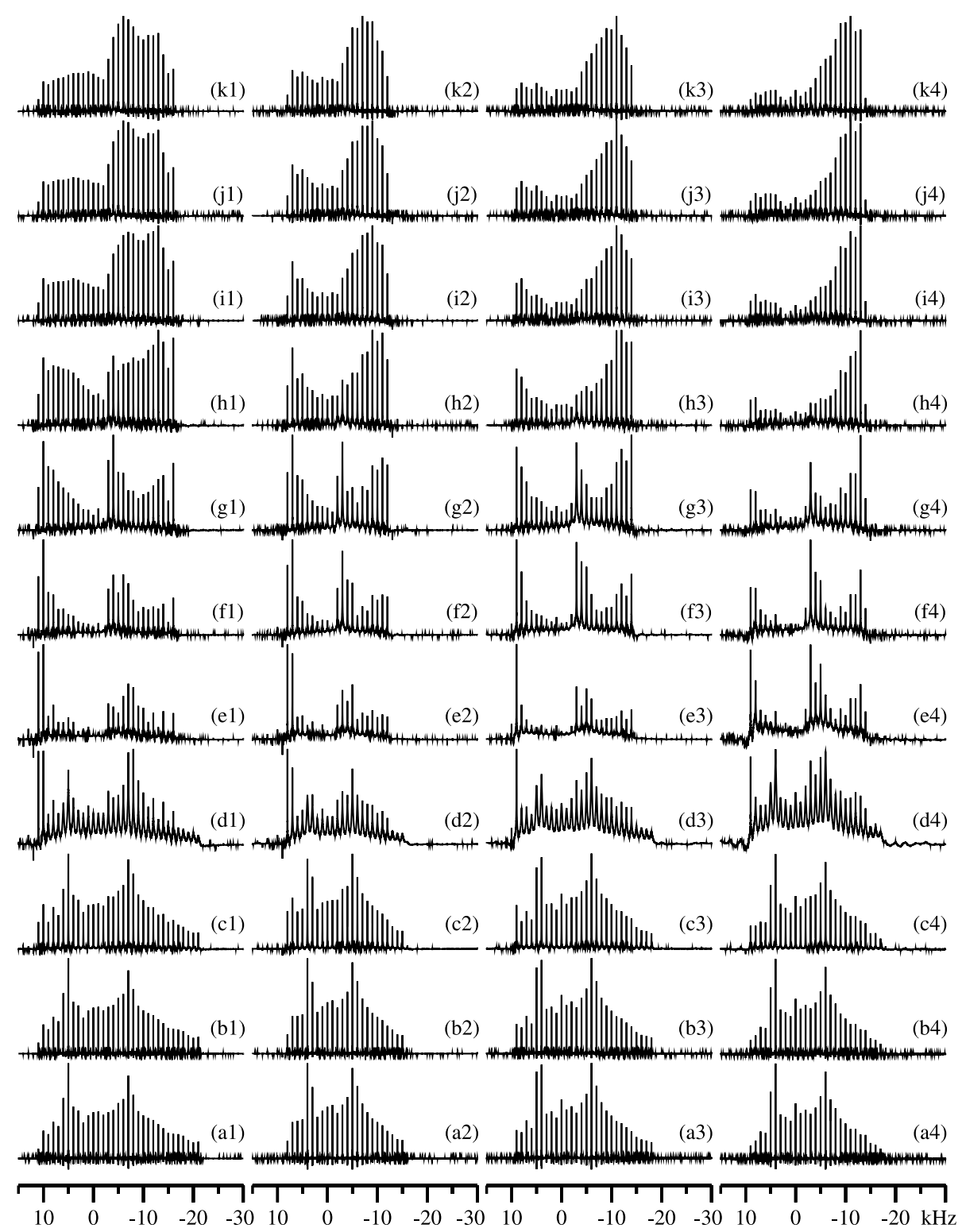

Fig. 2. Calculated static QCPMG spectra for a two-site jump employing rate constants of (a1, a2, a3, a4) $10^{0}$, (b1, b2, b3, b4) 10 ${ }^{1},(\mathrm{c} 1, \mathrm{c} 2, \mathrm{c} 3, \mathrm{c} 4) 10^{2}$, (d1, d2, d3, d4) $10^{3}$, (e1, e2, e3, e4) $10^{4}$, (f1,f2,f3,f4) $10^{5}$, (g1, g2, g3, g4) $10^{6}$, (h1,h2,h3,h4) 10 $10^{10} \mathrm{~Hz}$. In column 1 a series of spin-3/2 spectra are calculated employing $\omega_{\mathrm{rf}}=-31.25 \mathrm{kHz}, C_{Q}=2.0 \mathrm{MHz}$. The relative intensity of the spectra are (a1) 0.98 , (b1) 0.81 , (c1) 0.25 , (d1) 0.042, (e1) 0.061, (f1) 0.13, (g1) 0.19, (h1) 0.27, (i1) 0.48, (j1) 0.61, and (k1) 0.71, respectively. In column 2 a series of spin-5/2 spectra are calculated employing $\omega_{\mathrm{rf}}=-25.00 \mathrm{kHz}$ and $C_{Q}=4.0 \mathrm{MHz}$. The relative intensity of the spectra are (a2) 0.98 , (b2) 0.81 , (c2) 0.26, (d2) 0.055, (e2) 0.070, (f2) 0.12, (g2) 0.18, (h2) 0.29, (i2) 0.48, (j2) 0.59, and (k2) 0.73 , respectively. In column 3 a series of spin-7/2 spectra are calculated employing $\omega_{\mathrm{rf}}=-20.08 \mathrm{kHz}$ and $C_{Q}=7.0 \mathrm{MHz}$. The relative intensity of the spectra are (a3) $0.98,(\mathrm{~b} 3) 0.81$, (c3) 0.26 , (d3) 0.042 , (e3) 0.058, (f3) 0.083, (g3) 0.13 , (h3) 0.26 , (i3) 0.43 , (j3) 0.58 , and (k3) 0.73 , respectively. In column 4 a series of spin-9/2 spectra are calculated employing $\omega_{\mathrm{rf}}=-16.67 \mathrm{kHz}$ and $C_{Q}=10.0 \mathrm{MHz}$. The relative intensity of the spectra are (a4) 0.98, (b4) 0.81, (c4) 0.26 , (d4) 0.032 , (e4) 0.033 , (f4) 0.079 , (g4) 0.14 , (h4) 0.27 , (i4) 0.37 , (j4) 0.49 , and (k4) 0.60 , respectively. The intensities of the spectra corresponding to $k=10^{-9} \mathrm{~Hz}$ (not shown) are set to 1.0 for reference.

with spin-echo sideband separations of 1 (solid line), 5 (filled circles), and 10 (filled triangles) $\mathrm{kHz}$. These intensities follow similar patterns but the wider spin-echo sideband separation causes a steeper descend toward the minimum and a slower increase in intensity after the minimum. It must be stressed that the intensity in the spectra with a spin-echo sideband separation of $10 \mathrm{kHz}$ is 10.9 times higher than the ones with a separa- tion of $1 \mathrm{kHz}$ which makes the spectra with the larger separation very attractive for an initial series of quick VT experiments to obtain an overview of the dynamics in an unknown compound.

To explore the combined effect of the CSA- and the EFG-tensors a series of spin-7/2 QCPMG spectra have been calculated as both interactions may be large for this nucleus. In Fig. 4C the maximum intensities for 


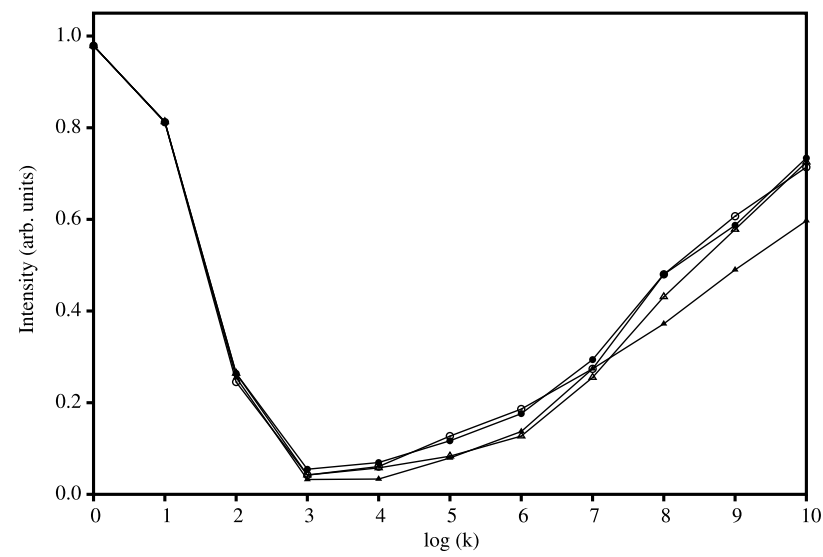

Fig. 3. Maximum intensities of calculated spin-3/2 (open circles), spin$5 / 2$ (filled circles), spin-7/2 (open triangles), and spin- $9 / 2$ (filled triangles) QCPMG spectra depicted in Fig. 2. The intensity of the spectra corresponding to $k=10^{-9} \mathrm{~Hz}$ for each nucleus are set to 1.0 for reference.

spectra without (solid line) and with the CSA interaction (filled circles) are displayed. The third intensity curve (filled triangles) illustrates the course of intensity if all spectra are normalized relative to the spectrum with quadrupolar interaction only and $k=10^{-9} \mathrm{~Hz}$ to show the effect of the large CSA of $500 \mathrm{ppm}$. In the slow motion range the course of intensity is similar for the two types of spectra but in fast limit the intensity of the spectra with the CSA interaction increases much faster and reaches $142 \%$ of its initial magnitude in the fast limit compared to $89 \%$ when no CSA is present. Hereby the intensity curve will be an easy way to tell if significant CSA is present.

\subsection{3-, 4-, and 6-site jumps}

Often 3-, 4-, and 6-site jumps are of interest as well and therefore series of QCPMG spectra for a spin-3/2 nucleus are calculated for these types of dynamic processes. The spectra are displayed in Fig. 5. Compared to the linebroadening effects observed for two-site jumps the effects on these spectra are more dramatic. Most pronounced are the linebroadenings in the regime $10^{3} \leqslant k \leqslant 10^{5} \mathrm{~Hz}$ (Figs. 5, d1-f1 and d2-f2) where spin-echo sideband almost collapses into a spin-echo lineshape. This broadening effect is strongest for the 6site jump and decreases with the order of the jumping process. In this context it is also noted that the linebroadening effect is observed for $k=10^{1} \mathrm{~Hz}$ for a 6 -site jump whereas it is not observed until $k=10^{2} \mathrm{~Hz}$ for 3and 4-site jumps. This also demonstrates that in the slow motion limit the linewidth of the spin-echo sidebands reflects the inverse lifetime in a site. In this limit identical spectra are expected for the same value of $N \cdot k$, where $N$ is the number of sites. In the fast regime the line narrowing sets in around $k=10^{8} \mathrm{~Hz}$ for all three types of
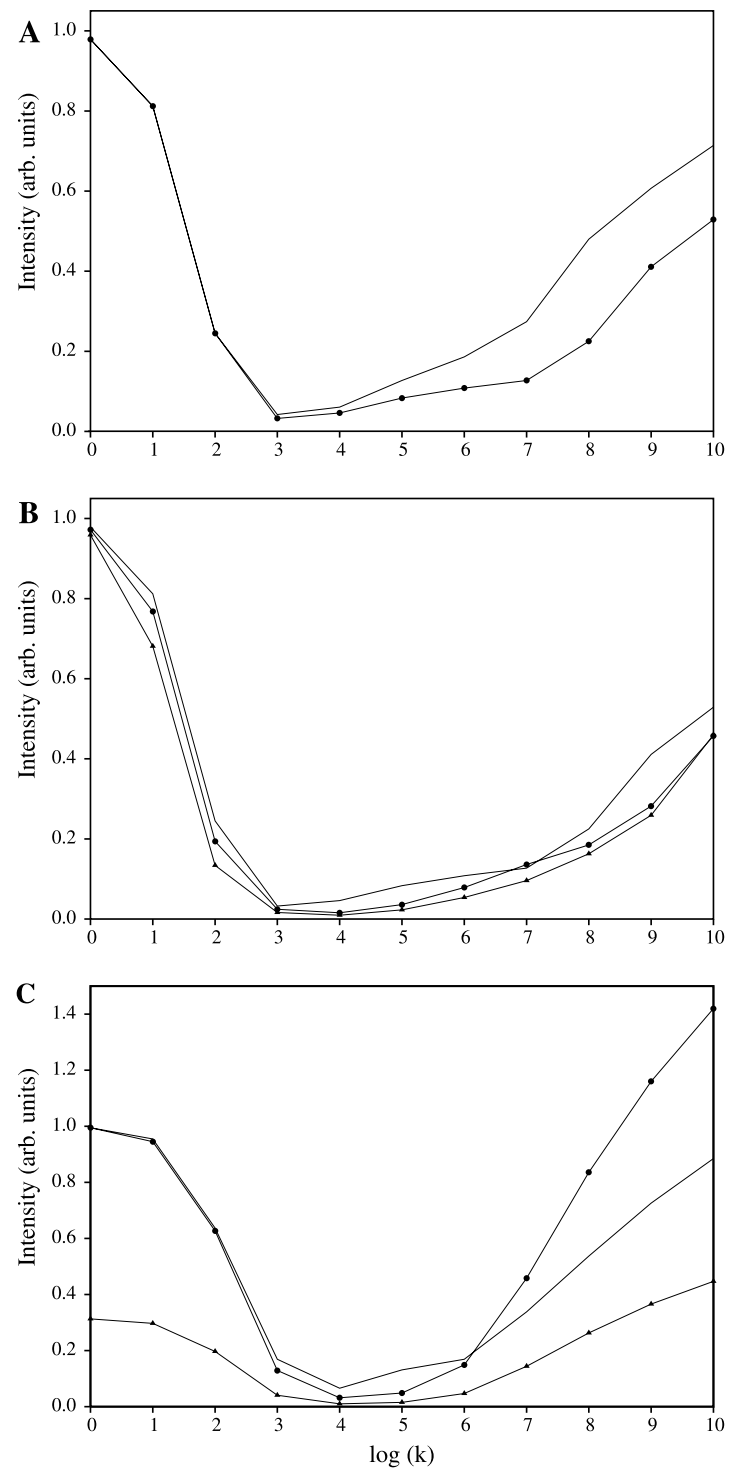

Fig. 4. Maximum intensities of calculated QCPMG spectra as a function of kinetic rate constant for a two-site jump. The intensities of the spectra corresponding to $k=10^{-9} \mathrm{~Hz}$ are set to 1.0 for reference. In (A) intensities corresponding to spin-3/2 QCPMG spectra calculated for $C_{Q}=2.0 \mathrm{MHz}$ (solid line) employing $\omega_{\mathrm{rf}}=-31.25 \mathrm{kHz}$ and $C_{Q}=4.0 \mathrm{MHz}$ using $\omega_{\mathrm{rf}}=-62.50 \mathrm{kHz}$ (filled circles) are displayed. In (B) maximum intensities corresponding to spin-3/2 QCPMG spectra calculated for $C_{Q}=4.0 \mathrm{MHz}$ using $\omega_{\mathrm{rf}}=-62.50 \mathrm{kHz}$ and spin-echo side band separations of $1 \mathrm{kHz}$ (solid line), $5 \mathrm{kHz}$ (filled circle, dwell time of $2.0 \mu \mathrm{s}, M=156$ and $\tau_{a}=200 \mu \mathrm{s}$ ), $10 \mathrm{kHz}$ (filled triangle, dwell time of $2.0 \mu \mathrm{s}, M=312$ and $\left.\tau_{a}=100 \mu \mathrm{s}\right)$, respectively, are displayed. In (C) maximum intensities of spin-7/2 QCPMG spectra calculated using $\omega_{\mathrm{rf}}=-50.0 \mathrm{kHz}$, $C_{Q}=15.0 \mathrm{MHz}, \quad \tau_{a}=\tau_{d}=200 \mu \mathrm{s}, \quad M=40, \quad \tau_{1}=\tau_{2}=\tau_{3}=\tau_{4}=$ $40.0 \mu \mathrm{s}$, dwell time $=1.0 \mu \mathrm{s}$, using a Gaussian linebroadening of $50 \mathrm{~Hz}$ are displayed (solid line). The line with filled circles displays the maximum intensity as a function of the kinetic rate constant when an additional CSA-interaction characterized by $\delta_{\sigma}=500 \mathrm{ppm}, \eta_{\sigma}=0.5$ with a relative orientation of the CSA and EFG-tensors are defined by the Euler angles $\Omega_{P P}^{\sigma}=\left(\frac{\pi}{2}, \frac{\pi}{6}, \frac{\pi}{2}\right)$ is included. The line with filled triangles illustrates the maximum intensities of the calculations with the CSA included relative to the spectrum with no CSA and $k=10^{-9} \mathrm{~Hz}$. 


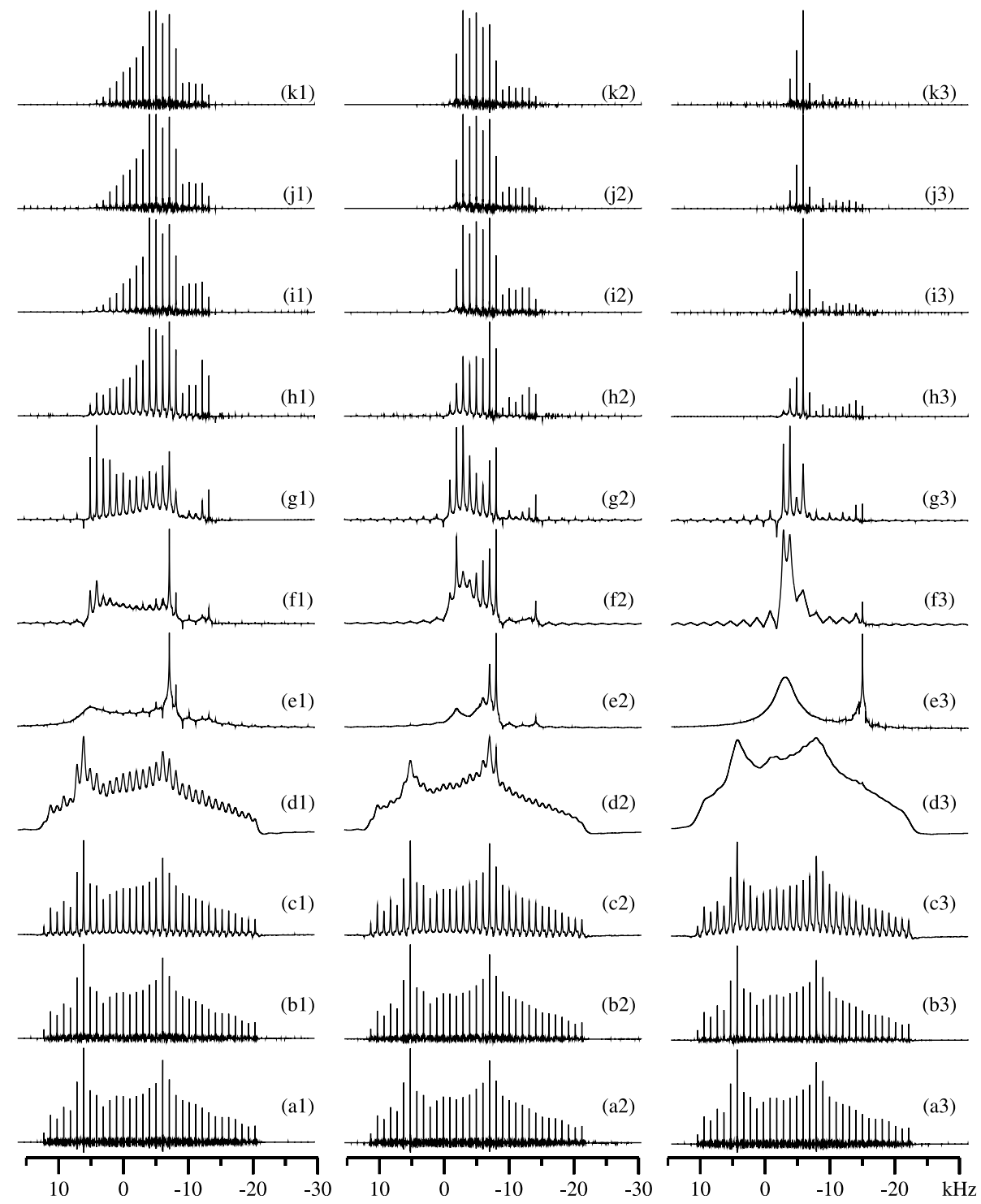

Fig. 5. Calculated static spin-3/2 QCPMG spectra for a 3-site (column 1), 4-site (column 2), and 6-site (column 3) jumps employing rate constants of (a1, a2, a3) $10^{0}$, (b1, b2, b3) $10^{1}$, (c1,c2, c3) $10^{2}$, (d1, d2, d3) $10^{3}$, (e1,e2, e3) $10^{4}$, (f1, f2,f3) $10^{5},(\mathrm{~g} 1, \mathrm{~g} 2, \mathrm{~g} 3) 10^{6},(\mathrm{~h} 1, \mathrm{~h} 2, \mathrm{~h} 3) 10^{7},(\mathrm{i} 1, \mathrm{i} 2, \mathrm{i} 3) 10^{8},(\mathrm{j} 1, \mathrm{j} 2, \mathrm{j} 3)$ $10^{9}$, and $(\mathrm{k} 1, \mathrm{k} 2, \mathrm{k} 3) 10^{10} \mathrm{~Hz}$. All spectra are calculated employing $\omega_{\mathrm{rf}}=-31.25 \mathrm{kHz}$ and $C_{Q}=2.0 \mathrm{MHz}$. In column 1 the relative intensity of the spectra are (a1) 0.96, (b1) 0.68, (c1) 0.13, (d1) 0.014, (e1) 0.0061, (f1) 0.030, (g1) 0.088, (h1) 0.18, (i1) 0.60, (j1) 0.81, and (k1) 1.07, respectively. In column 2 the relative intensity of the spectra are (a2) 0.94 , (b2) 0.58 , (c2) 0.089 , (d2) 0.012 , (e2) 0.0094, (f2) 0.032 , (g2) 0.14 , (h2) 0.38 , (i2) 0.72 , (j2) 0.94 , and (k2) 1.29 , respectively. In column 3 the relative intensity of the spectra are (a3) $0.90,(\mathrm{~b} 3) 0.43$, (c3) 0.053 , (d3) $0.0053,(\mathrm{e} 3) 0.0014,(\mathrm{f} 3) 0.038$, (g3) 0.23 , (h3) 0.80 , (i3) 2.13, (j3) 2.67, and (k3) 3.39, respectively. The intensities of the spectra corresponding to $k=10^{-9} \mathrm{~Hz}$ (not shown) are set to 1.0 for reference.

motion. For all three processes the width of the central transition becomes narrower in the limit of fast dynamics.

The corresponding maximum intensity curves are displayed in Fig.6. For slow dynamics the intensities for the higher order jumps decrease faster but after the broad minimum between $k=10^{3}$ and $k=10^{5} \mathrm{~Hz}$ they also increase much faster. Especially for the 6-site jump where the intensity in the fast limit is $339 \%$ of the initial intensity. For the 3-and 4-site jumps the intensities in the fast limit are 107 and $129 \%$ of the initial intensity, respectively.

\subsection{Effect of $M A S$}

When investigating dynamics of ${ }^{2} \mathrm{H}$ by NMR the single pulse MAS experiment has been demonstrated to be extremely sensitive towards the kinetic rate constant [12,31]. In ${ }^{2} \mathrm{H}$ spectra QCPMG and single-pulse MAS experiments both split the static lineshape into sidebands whose lineshape are highly dependent on dynamics. For the central transition the single-pulse MAS experiment only narrows the lineshape [13] but besides that it does not provide any more information than the QE experiment. Therefore the ability to produce 


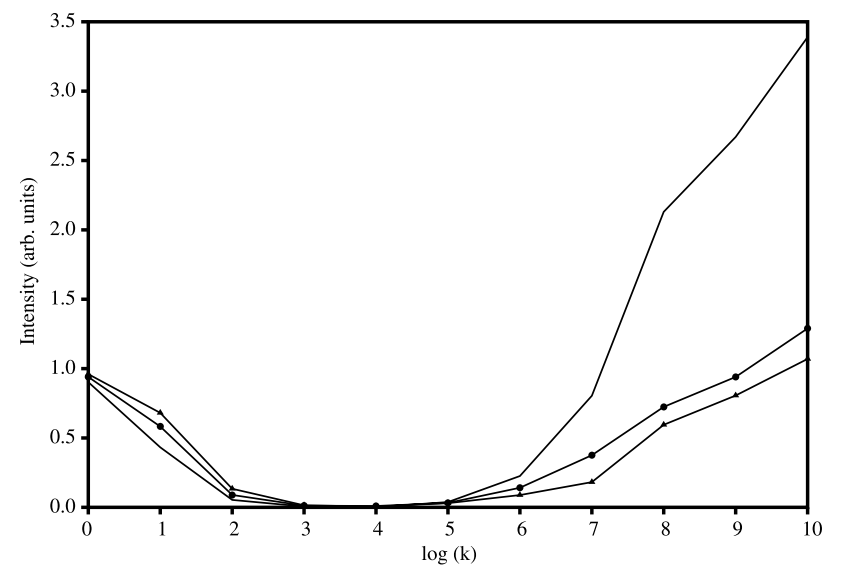

Fig. 6. Maximum intensities of calculated spin-3/2 QCPMG spectra depicted in Fig. 5 for 3-site (filled triangles), 4-site (filled circles) and 6site (solid line) jump processes. The intensities of the spectra corresponding to $k=10^{-9} \mathrm{~Hz}$ are set to 1.0 for reference.

sidebands that are sensitive to dynamics is unique for the QCPMG experiment. The combination of MAS and QCPMG may still provide interesting information in a addition to the one obtained from the static
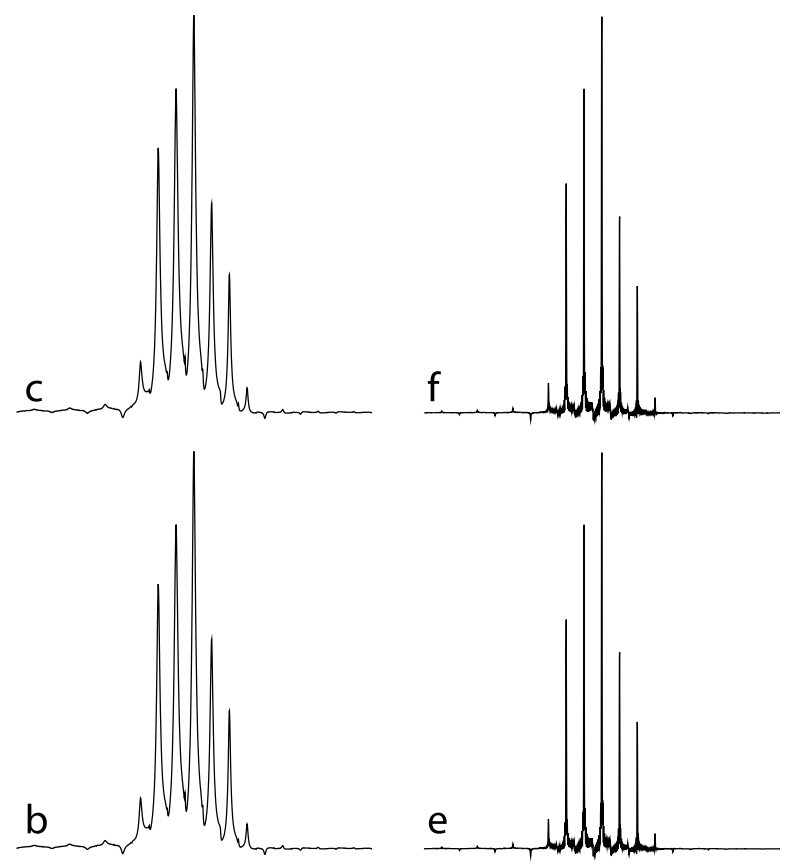

a

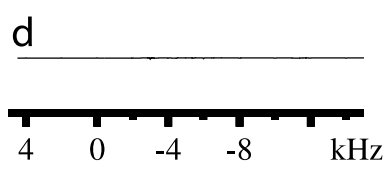

Fig. 7. Calculated spin-3/2 QCPMG-MAS spectra for a two-site jump employing time increments of (c,f) $1.0 \mu \mathrm{s}$ and (b,e) $0.1 \mu$ s using rate constants of (b,c) $10^{6}$, and (e,f) $10^{9} \mathrm{~Hz}$. All spectra are calculated for $\omega_{\mathrm{rf}}=-31.25 \mathrm{kHz}$ and $C_{Q}=2.0 \mathrm{MHz}$. The spectrum in (a) is the difference between (c) and (b), and the spectrum in (d) is the difference between (f) and (e). Both difference spectra are scaled vertically by a factor of 10 .
QCPMG experiment. Therefore the effects of the QCPMG-MAS experiment have been explored in the following.

When MAS is included the Hamiltonian becomes time-dependent and it is important to propagate the density operator in sufficiently small time increments to ensure convergence. This is particular important during rf-pulses. In Fig. 7 calculated QCPMG-MAS spectra for a spin-3/2 nucleus performing a two-site jump and using time increments during the rf-pulses of $1.0 \mu$ s (Figs. 7c and f) and $0.1 \mu$ s (Figs. 7b and e),

$\mathbf{A}$
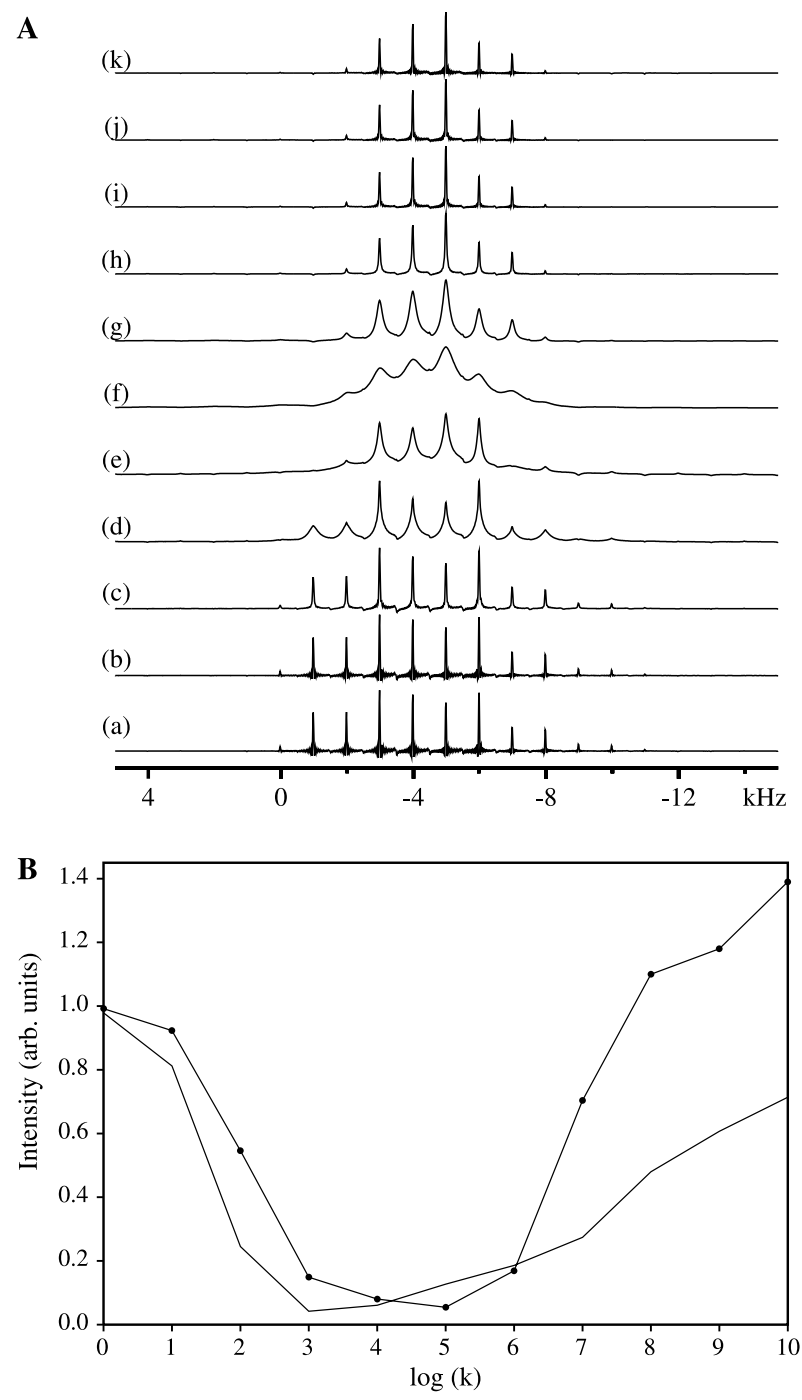

Fig. 8. (A) Calculated spin-3/2 QCPMG-MAS spectra for a 2-site jump employing rate constants of (a) $10^{0}$, (b) $10^{1}$, (c) $10^{2}$, (d) $10^{3}$, (e) $10^{4}$, (f) $10^{5}$, (g) $10^{6}$, (h) $10^{7}$, (i) $10^{8}$, (j) $10^{9}$, and (k) $10^{10} \mathrm{~Hz}$. All spectra are calculated employing $\omega_{\mathrm{rf}}=-31.25 \mathrm{kHz}$ and $C_{Q}=2.0 \mathrm{MHz}$. The relative intensity of the spectra are (a) 0.99 , (b) 0.92 , (c) 0.55 , (d) 0.15 , (e) 0.080 , (f) 0.055 , (g) 0.17, (h) 0.70, (i) 1.10, (j) 1.18, and (k) 1.39, respectively. (B) Maximum intensities of spin-3/2 QCPMG-MAS (filled circles) and static spin-3/2 QCPMG (solid line) spectra. The intensities of the spectra corresponding to $k=10^{-9} \mathrm{~Hz}$ (not shown) are set to 1.0 for reference. 
respectively, and their difference spectra (Figs. 7a and d) are displayed. The spectra are calculated for dynamic rate constants of $10^{6} \mathrm{~Hz}$ (Figs. $7 \mathrm{~b}$ and c) and $10^{9} \mathrm{~Hz}$ (Figs. 7e and f). As the difference spectra even vertically by a factor of 10 are very close to zero intensity it is concluded that time increments of $1.0 \mu \mathrm{s}$ is sufficient to ensure convergence during rf-pulses. This time increment will also be used during periods of free precession.

Fig. 8A displays a series of QCPMG-MAS spectra for a spin-3/2 nucleus performing a two-site jump under $20 \mathrm{kHz}$ of MAS. As for the static spectra the spin-echo sidebands are narrow in the slow motion limit below $k=10^{2} \mathrm{~Hz}$ (Fig. 8A, a and b) and in the fast motion limit above $k=10^{8} \mathrm{~Hz}$ (Fig. 8A, $\mathrm{j}$ and $\mathrm{k}$ ). In the intermediate region the spin-echo sidebands become broader up to $k=10^{5} \mathrm{~Hz}$ (Fig. 8A, f) and then narrows up for faster dynamics. At $k=10^{5} \mathrm{~Hz}$ the spin-echo sidebands are almost collapsed into a spin-echo lineshape. As for the static QCPMG spectra the lineshape becomes narrower in the fast limit regime but the width of the spin-echo sidebands goes through a maximum around $k=10^{5} \mathrm{~Hz}$ in the process of narrowing up the lineshape which is not observed in the static QCPMG spectra.

In Fig. 8B the maximum intensities of the QCPMG and QCPMG-MAS spectra are depicted. One striking difference is the very clear minimum range for the QCPMG-MAS spectra between $k=10^{3}$ and $k=10^{6} \mathrm{~Hz}$. The intensity for the static spectra slowly increases and reaches around $75 \%$ of the initial intensity at $k=10^{10} \mathrm{~Hz}$ whereas the intensity increase is much steeper under MAS and reaches $139 \%$ of the initial intensity in the fast limit.

\section{Conclusions}

In the present work the effects of molecular dynamics in QCPMG type solid-state NMR spectra have been analyzed theoretically and numerically. Two-site jump processes have been analyzed in particular and it has been shown that significant linebroadening of the spinecho sidebands is induced in when the kinetic rate constant $(k)$ is in the region $10^{2} \leqslant k \leqslant 10^{7} \mathrm{~Hz}$ - particular in the $10^{3} \leqslant k \leqslant 10^{5} \mathrm{~Hz}$ regime. For higher order jump processes, 3-, 4-, and 6-site jumps, the linebroadening effect is even more pronounced. Therefore the QCPMG approach is an attractive alternative to the standard QE experiment as the spin-echo sidebands are very sensitive to $k$ and the maximum intensity is about an order of magnitude higher.

Based on the present numerical results it is anticipated that QCPMG and QCPMG-MAS experiments will be important tools for analysis of molecular dynamics by solid-state NMR spectroscopy on half-integer quadrupolar nuclei.

\section{Acknowledgment}

This research was supported by grants from the Biotechnology Initiative of the Danish Research Councils.

\section{References}

[1] E.A. Behrens, D.M. Poojary, A. Clearfield, Syntheses, X-ray powder structures, and preliminary ion-exchange properties of germanium-substituted titanosilicate pharmacosiderites: $\mathrm{HM}_{3}(\mathrm{AO})_{4}\left(\mathrm{BO}_{4}\right)_{3} \cdot 4 \mathrm{H}_{2} \mathrm{O}(\mathrm{M}=\mathrm{K}, \mathrm{Rb}, \mathrm{Cs} ; \mathrm{A}=\mathrm{Ti}, \mathrm{Ge} ; \mathrm{B}=\mathrm{Si}$, Ge, Chem. Mater. 10 (1998) 959-967.

[2] M. Nyman, F. Bonhomme, R.S. Maxwell, T.M. Nenoff, First Rb silicotitanate phase and its $\mathrm{K}$-structural analogue: new members of the SNL-A family $\left(\mathrm{Cc}-\mathrm{A}_{2} \mathrm{TiSi}_{6} \mathrm{O}_{15} ; \mathrm{A}=\mathrm{K}, \mathrm{Rb}, \mathrm{Cs}\right)$, Chem. Mater. 13 (2001) 4603-4611.

[3] P.D. Southon, R.F. Howe, Spectroscopic studies of disorder in the microporous titanosilicate ETS-10, Chem. Mater. 14 (2002) 4209-4218.

[4] L-S. Du, J.F. Stebbins, Solid-state NMR study of metastable immiscibility in alkali borosilicate glasses, J. Non-Cryst. Sol. 315 (2003) 239-255.

[5] S. Sham, G. Wu, Solid-state ${ }^{25} \mathrm{Mg}$ NMR study of inner-sphere $\mathrm{Mg}^{2+}$ binding complexes, Inorg. Chem. 39 (2000) 4-5.

[6] C.V. Grant, V. Frydman, L. Frydman, Solid-state ${ }^{25} \mathrm{Mg}$ NMR of a magnesium(II) adensosine $5^{\prime}$-triphosphate complex, J. Am. Chem. Soc. 122 (2001) 11743-11744.

[7] A.S. Lipton, T.A. Wright, M.K. Bowman, D.L. Reger, P.D. Ellis, Solid-state ${ }^{67} \mathrm{Zn}$ NMR spectroscopy in bioinorganic chemistry. spectra of four- and six-coordinate zinc pyrazolylborate complexes obtained by management of proton relaxation rates with a paramagnetic dopant, J. Am. Chem. Soc. 124 (2002) 58505860 .

[8] A.S. Lipton, R.W. Heck, P.D. Ellis, Zinc solid-state NMR spectroscopy of human carbonic anhydrase: implications for the enzymatic mechanism, J. Am. Chem. Soc. 126 (2004) 47354739.

[9] S. Kaufmann, S. Wefing, D. Schaefer, H.W. Spiess, Twodimensional exchange nuclear magnetic resonance of powder samples. III. Transition to motional averaging and application to the glass transition, J. Chem. Phys. 93 (1990) 197-214.

[10] A.E. Aliev, K.D.M. Harris, I.J. Shannon, C. Glidwell, C.M. Zakaria, P.A. Schofield, Solid-state ${ }^{2} \mathrm{H}$ and ${ }^{13} \mathrm{C}$ NMR studies of hydrogen-bond dynamics in ferrocene-1,1'-diylbis(diphenylmethanol), J. Phys. Chem. 99 (1995) 12008-12015.

[11] V. Gérardy-Montouillout, C. Malveau, P. Tekely, Z. Olender, Z. Luz, ODESSA, a new 1D NMR exchange experiment for chemically equivalent nuclei in rotating solids, J. Magn. Reson. A 123 (1996) 7-15.

[12] J.H. Kristensen, G.L. Hoatson, R.L. Vold, Effects of restricted rotational diffusion on ${ }^{2} \mathrm{H}$ magic angle spinning nuclear magnetic resonance spectra, J. Chem. Phys. 110 (1999) 4533-4553.

[13] J.H. Kristensen, I. Farnan, Measurement of molecular motion in solids by nuclear magnetic resonance spectroscopy of halfinteger quadrupole nuclei, J. Chem. Phys. 114 (2001) 96089624.

[14] J.H. Kristensen, I. Farnan, Anisotropic relaxation and motion of half-integer quadrupole nuclei studied by central transition nuclear magnetic resonance spectroscopy, J. Magn. Reson. 158 (2002) 99-125.

[15] M. Witschas, H. Eckert, H. Freiheit, A. Putnis, G. Korus, M. Jansen, Anion rotation and cation diffusion in low-temperature sodium orthophosphate: results from solid-state NMR, J. Phys. Chem. A. 105 (2001) 6808-6816. 
[16] R.W. Schurko, S. Wi, L. Frydman, Dynamic effects on the powder line shapes of half-integer quadrupolar nuclei: a solidstate NMR study of $\mathrm{XO}_{4}^{-}$groups, J. Phys. Chem. A. 106 (2002) 51-62.

[17] F.H. Larsen, H.J. Jakobsen, P.D. Ellis, N.C. Nielsen, Molecular dynamics from ${ }^{2} \mathrm{H}$ quadrupolar Carr-Purcell-Meiboom-Gill solid-state NMR spectroscopy, Chem. Phys. Lett. 292 (1998) 467473.

[18] F.H. Larsen, H.J. Jakobsen, P.D. Ellis, N.C. Nielsen, Sensitivityenhanced quadrupolar-echo NMR of half-integer quadrupolar nuclei. Magnitudes and relative orientation of chemical shielding and quadrupolar coupling tensors, J. Phys. Chem. A 101 (1997) 8597-8606.

[19] F.H. Larsen, H.J. Jakobsen, P.D. Ellis, N.C. Nielsen, QCPMGMAS NMR of half-integer quadrupolar nuclei, J. Magn. Reson. 131 (1998) 144-147.

[20] F.H. Larsen, H.J. Jakobsen, P.D. Ellis, N.C. Nielsen, High-field QCPMG-MAS NMR of half-integer nuclei quadrupolar nuclei with large quadrupole couplings, Mol. Phys. 95 (1998) 11851195.

[21] T.M. Barbara, M.S. Greenfield, R.L. Vold, R.R. Vold, Deuterium quadrupole echo NMR spectroscopy. I. Effects of chemical exchange during single and composite pulses, J. Magn. Reson. 69 (1986) 311-330.

[22] F.J. Dyson, The radiation theories of Tomonaga, Schwinger, and Feynman, Phys. Rev. 75 (1949) 486-502.
[23] G.J. Bowden, W.D. Hutchison, Tensor operator formalism for multiple-quantum NMR. 1. Spin-1 nuclei, J. Magn. Reson. 67 (1986) 403-414.

[24] G.J. Bowden, W.D. Hutchison, J. Khachan, Tensor operator formalism for multiple-quantum NMR. 2. Spins $3 / 2,2$ and $5 / 2$ and general I, J. Magn. Reson. 67 (1986) 415-437.

[25] D.M. Brink, G.R. Satchler, Angular Momentum, Clarendon Press, Oxford, 1993.

[26] M.S. Greenfield, A.D. Ronemus, R.L. Vold, R.R. Vold, P.D. Ellis, T.E. Raidy, Deuterium quadrupole-echo NMR spectroscopy. III. Practical aspects of lineshape calculations for multiaxis rotational processes, J. Magn. Reson. 72 (1987) 89-107.

[27] S.K. Zaremba, Good Lattice points, discrepancy, and numerical integration, Ann. Mate. Pura Appl. 73 (1966) 293-317.

[28] E. Anderson, Z. Bai, C. Bischof, S. Blackford, J. Demmel, J. Dongarra, J. Du Croz, A. Greenbaum, S. Hammarling, A. McKenney, D. Sorensen, LAPACK Users' Guide, Society for Industrial and Applied Mathematics, Philadelphia, PA, 1999. Available from: $<$ http://www.netlib.org/lapack/ $>$.

[29] I. Solomon, Multiple echoes in solids, Phys. Rev. 110 (1958) 6165.

[30] I.D. Weisman, L. Bennett, Quadrupolar echoes in solids, Phys. Rev. 181 (1969) 1341-1350.

[31] J.H. Kristensen, H. Bildsøe, H.J. Jakobsen, N.C. Nielsen, Theory and simulations of molecular dynamics in ${ }^{2} \mathrm{H}$ MAS NMR, J. Magn. Reson. 100 (1992) 437-443. 